\title{
Virtual surgical planning of inferior alveolar nerve microsurgery and benign pathology of the mandible: case reports
}

\author{
Christina Gory^, Vincent Ziccardi \\ Department of Oral and Maxillofacial Surgery, Rutgers School of Dental Medicine, Newark, NJ, USA \\ Correspondence to: Christina Gory, DDS, Resident, Department of Oral and Maxillofacial Surgery, Rutgers School of Dental Medicine, 110 Bergen \\ Street Room B854 Newark, NJ 07103-2400, USA. Email: clg180@sdm.rutgers.edu.
}

\begin{abstract}
With the aid of virtual surgical planning (VSP) software as well as 3-dimensional printing technology, custom surgical guides can be created utilizing the pre-operative data of patients, including both computed tomography and intraoral scans. VSP technology allows for the customization of surgical guides which can provide an array of benefits that aid in improving patient care. Surgical guides can be designed as occlusal borne, bone borne, or a combination of the two. Though an intraoral scan is beneficial to bolster surgical guide accuracy, it is not necessary, as demonstrated in the following case presentations. This manuscript will demonstrate the use of custom occlusal and bone borne surgical guides that were used for access to the inferior alveolar nerve for exploratory microsurgery as well as two cases necessitating removal of benign pathology within the mandible. Surgical guides aid in improving intraoperative efficiency, in decreasing operating time and in reducing potential surgical trauma. Overall, we expect a decrease in morbidity due to the more predictable nature of guided surgeries. The use of custom surgical guides allows for a more precise and conservative osteotomy, thereby decreasing morbidity. The more efficient and predictive nature of guided surgery aids in patient recovery and surgical outcomes, as well.
\end{abstract}

Keywords: Guided surgery; inferior alveolar nerve; microsurgery; benign pathology; case reports

Received: 15 July 2021; Accepted: 13 October 2021; Published: 10 September 2022.

doi: $10.21037 /$ fomm-21-84

View this article at: https://dx.doi.org/10.21037/fomm-21-84

\section{Introduction}

With the aid of virtual surgical planning (VSP) software and 3D printing technology, custom surgical guides utilizing the data from patient's pre-operative imaging can be fabricated. A surgical guide can aid the surgeon in operating in a more controlled and predictable manner, especially important when vital anatomical structures are proximal to the operative field. Decreasing surgical trauma and morbidity is an additional potential benefit to the patient. Time under general anesthesia, which correlates to duration of surgical procedure, should ideally be minimized. This can be accomplished when procedures are performed as minimally invasive as possible. Care should be taken not to compromise clinical results. Additionally, minimizing surgical time in this manner can lead to improvement in patient's post-operative course and outcomes (1-3). Guided surgery can reduce surgical time and therefore time under general anesthesia and in the operating room and potentially overall surgical costs (1,2-6). Most importantly, custom surgical guides can play a crucial role in a procedure that involves vital structures and preservation of these structures may dictate surgical success $(1,7)$.

A custom surgical guide is helpful in accessing the inferior alveolar nerve (IAN) for microsurgery as well as accessing intrabony lesions of the mandible that have not perforated the buccal plate. Without a surgical guide designed based on the patient's imaging, these procedures

^ ORCID: 0000-0002-4252-692X. 


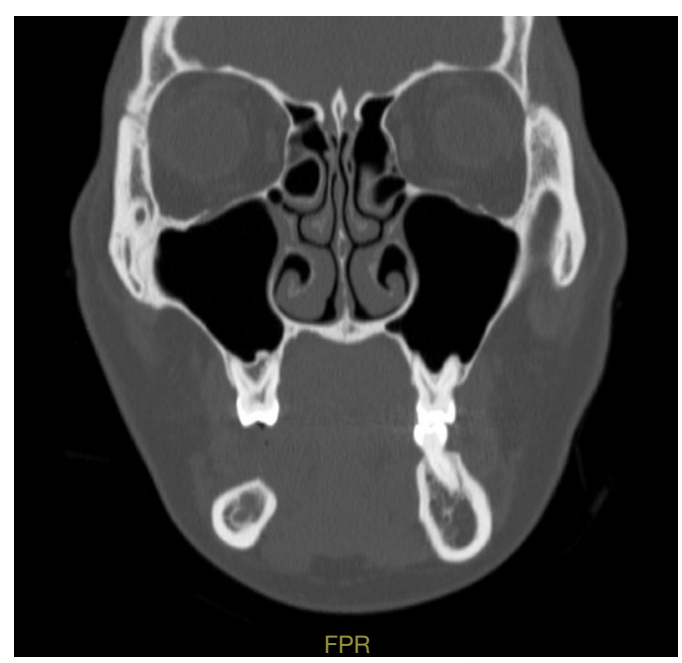

Figure 1 Coronal view of CT Maxillofacial exam demonstrating a disruption of continuity and decrease in cortication of the inferior alveolar nerve canal.

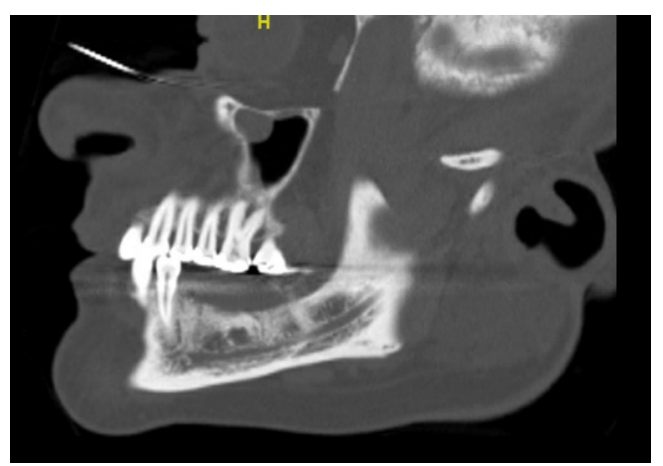

Figure 2 Sagittal oblique view of the right mandible revealing consolidating bone graft and constriction of the inferior alveolar nerve canal.

can involve large osteotomies and increased potential risks. In this series, the pre-operative data was used in designing custom cutting guides for all three cases presented and the workflows all followed a similar protocol. In this series, Stryker (Kalamazoo, MI) and 3D systems VSP (Littleton, CO, USA) was utilized, though this type of custom guide design is not overly sophisticated, thereby enabling the surgeon's preferred manufacturer to be used. CT maxillofacial exams are necessary while intraoral scans can be considered optional, though they bolster the fit of a guide that is designed to have an occlusal borne component. Without a surgical guide, a sagittal split osteotomy or large buccal osteotomy to localize the area of interest would be required to access the IAN or intrabony lesions of the mandible. Both techniques may involve more surgical trauma than necessary. We present the following article in accordance with the CARE reporting checklist (available at https://fomm.amegroups.com/article/view/10.21037/ fomm-21-84/rc).

\section{Case presentation 1}

A 58-year-old healthy female patient presented to the Rutgers Oral and Maxillofacial Surgery faculty practice with a chief complaint consistent with a right IAN injury. The patient had no relevant medical history and the review of systems was negative. Three months prior to her initial presentation, she had extractions of her lower right posterior teeth and allogeneic bone grafting. She reported feeling an "electrical-like" shock during the procedure and never regained sensation of her lip and chin after the local anesthesia dissipated. She experienced no subjective or objective improvement in sensation since initial surgery.

Baseline IAN testing was completed, including typical parameters of hot and cold sensation, two-point discrimination, brush, plastic filament detection, vibration and pin prick, which revealed a significant deficit consistent with the patient's reported history. A CT maxillofacial exam without contrast revealed an area of decreased cortication of the IAN canal with constriction, as well as the consolidating bone graft at site \#29-30, as appreciated in Figures 1 and 2. Neurosensory retraining exercises were reviewed with the patient and a daily over the counter B12 supplementation was recommended. The patient was followed for a total of three months, with diagnostic subjective nerve testing being performed at each appointment. The patient showed no signs of spontaneous improvement and had persistent lip biting, feeling of numbness and "pins and needles" sensation.

After six months passed since the date of the original injury and with no improvement in sensation, the patient elected to undergo exploratory IAN microsurgery. The STL files from an intraoral scan and DICOM files from a CT maxillofacial exam were utilized in a VSP session to create an occlusal and bone borne guide. The design of the surgical guide is demonstrated in Figure 3, which includes an arm extending inferiorly onto the body of the mandible with an osteotomy guide placed at the location of the suspected injury of IAN as noted on the pre-operative CT scan. Two temporary fixation holes were added to the superior aspect of the osteotomy guide to bolster stability 


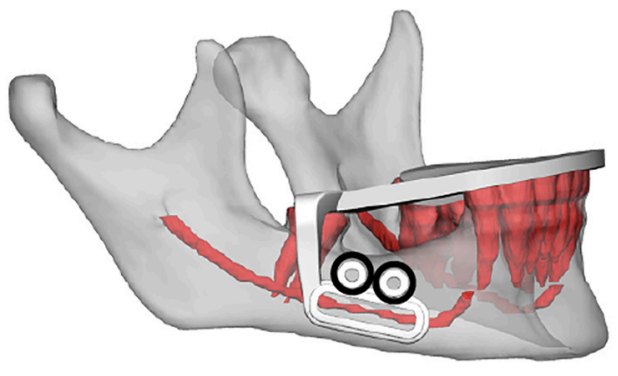

Figure 3 The occlusal and bone borne osteotomy guide mock up designed to aid in the accessing of the inferior alveolar nerve at the site of injury.

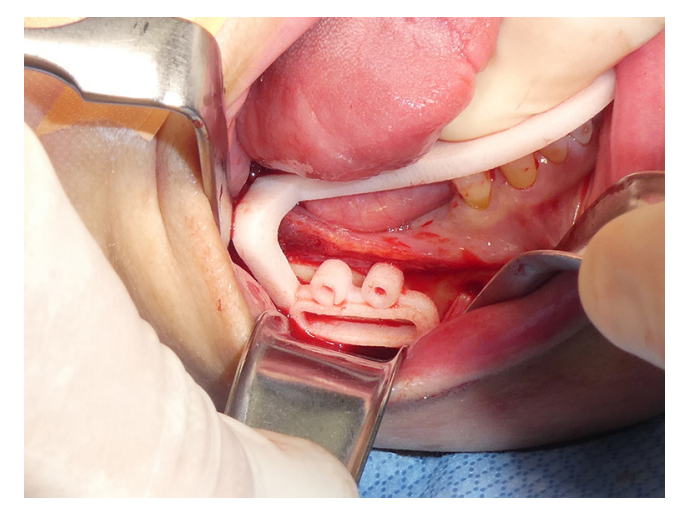

Figure 4 The seating of the surgical guide is appreciated with retraction of the mental nerve.

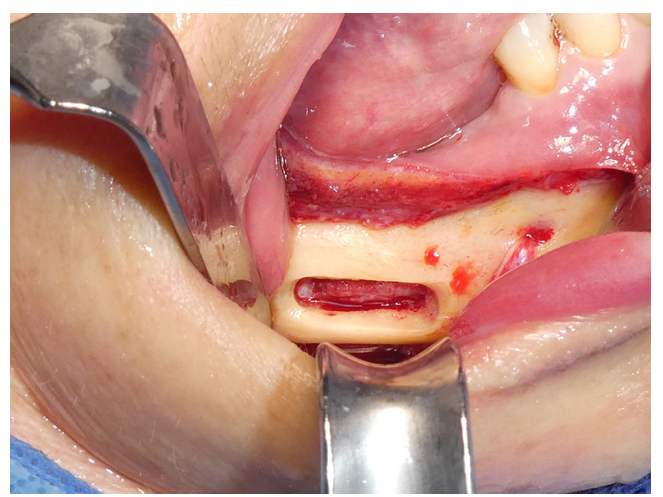

Figure 5 The osteotomy has been completed and the lateral window removed, thereby rendering the inferior alveolar nerve visible and accessible.

at the window in case there was any rocking of the guide intraoperatively, which would be more detrimental in this case due to the relatively small size of the surgical field.
The procedure was conducted under general anesthesia. The field was prepared and draped in a sterile manner and lidocaine 1\% 1:100,000 epinephrine was locally infiltrated. A 15 blade was utilized to create a papillary sparing incision and a full thickness mucoperiosteal flap was elevated. Care was taken to locate and dissect the mental nerve to prevent traction or direct injury. The cutting guide was placed intraorally and verified to be fully seated without any rocking making the fixation screws unnecessary (see Figure 4). A round bur was utilized to create a peripheral osteotomy within the window of the cutting guide, which was then removed and the osteotomy was deepened until the lateral window of cortical bone could be removed (Figure 5). Access to the IAN was then appreciated and the trigeminal nerve microsurgical procedure was completed without complications. The patient returned for a routine one week and one month follow up to assess acute healing. By one month mark, the incision site was very well healed, there was no evidence of acute infection or pathological fracture, and her opening had returned to normal limits. The postoperative panoramic image demonstrates a well demarcated ovate osteotomy window. Full sensory recovery results are still being monitored at the time of publication.

\section{Case presentation 2}

A 37-year-old female presented to the senior author for consultation for conservative treatment of a recurrent ameloblastoma of her right posterior mandible. The patient's medical history was unremarkable other than a recent caesarian section. She had previously undergone treatment for the initial and subsequent recurrences of ameloblastoma using conservative surgical approach with outside surgeons. As seen on the pre-operative panoramic image (Figure 6) and CT maxillofacial exam without contrast, the lesion's close relation with the IAN and mental nerve and foramen is appreciated (Figures 7-9). It is important to note that the patient declined the recommended treatment of resection, which would minimize chance of further recurrence. Clinically she had normal mandibular and masticatory function and she was missing teeth \#29,30,31, and 32. There was appreciation of intraoral scar bands from prior procedures, and there was mild expansion of the posterior right alveolar ridge. The patient denied any $\mathrm{V} 3$ paresthesia.

A CT maxillofacial exam demonstrated a $1.14 \mathrm{~cm} \times$ $0.69 \mathrm{~cm} \times 0.67 \mathrm{~cm}$ lesion that was intimately involved with the IAN. After discussion of all surgical options, the patient 


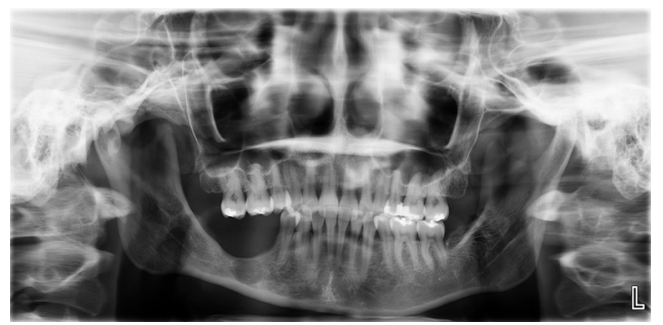

Figure 6 Panoramic image demonstrating the recurrent radiolucent lesion in the right mandibular body in close relationship with the inferior alveolar nerve.

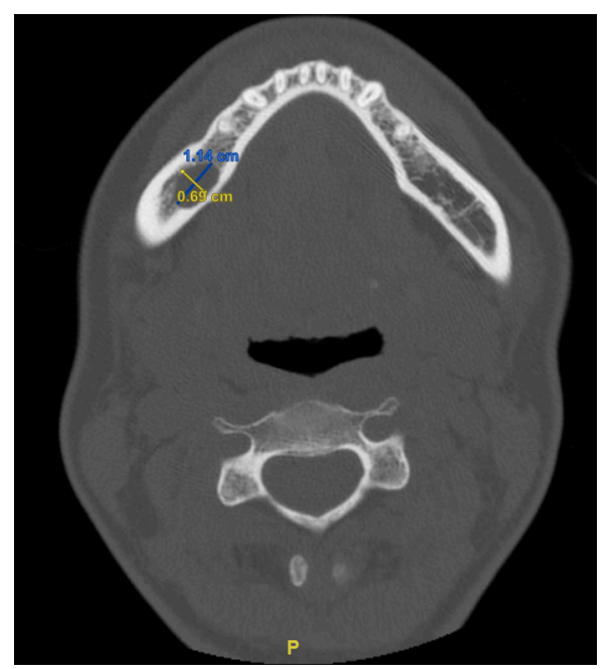

Figure 7 Axial view of the CT maxillofacial exam demonstrating the A-P dimensions of the recurrent ameloblastoma.

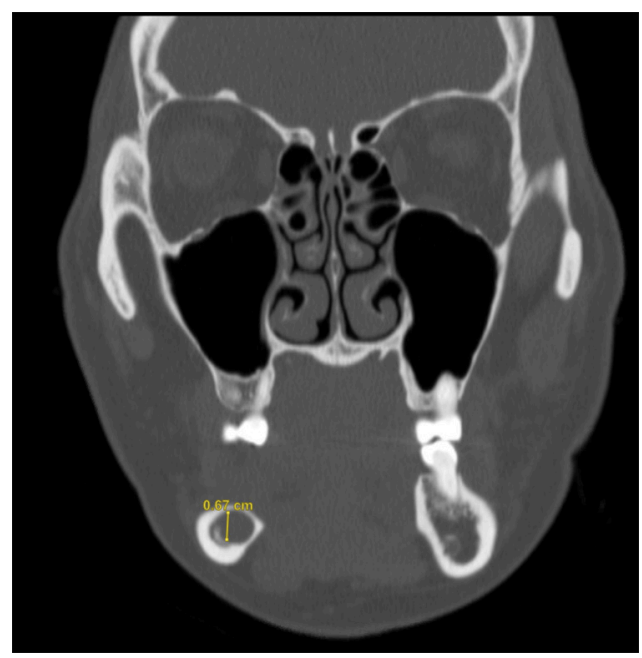

Figure 8 The coronal view demonstrates the height of the lesion of $0.67 \mathrm{~cm}$ within the atrophic right mandibular body.

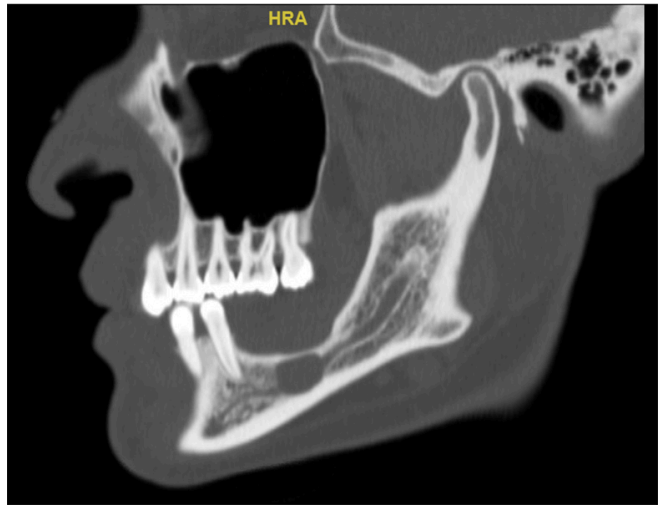

Figure 9 The close relationship with the recurrent ameloblastoma and the inferior alveolar nerve is appreciated in the sagittal view of the patient's preoperative CT.

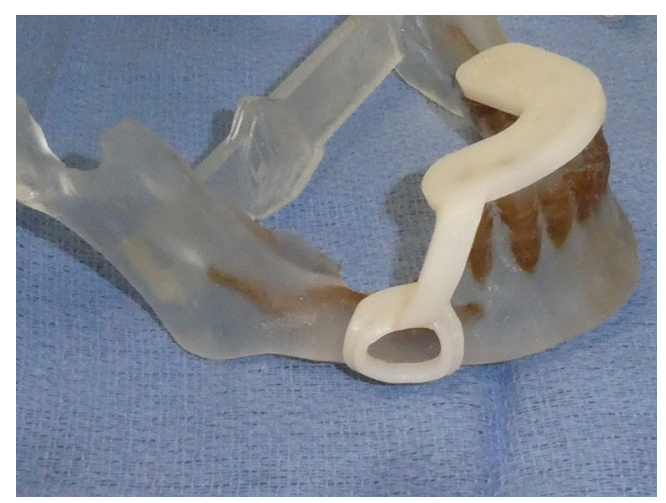

Figure 10 Occlusal and bone borne surgical osteotomy guide seated on the stereolithic model.

elected to proceed with enucleation of this lesion. Due to the location of the recurrent lesion, a custom surgical guide was fabricated to access the lesion within the mandibular body. The STL files from an intraoral scan and the DICOM data from the patient's CT maxillofacial exam were again utilized during a VSP session to design an occlusal and bone borne surgical guide that would allow the creation of a precise osteotomy corresponding to the location of the lesion, seen placed on the stereolithic model in Figure 10.

Intraoperatively, after the dissection was completed, the guide was inserted to ensure there was adequate flap elevation and that was seating passively and without rocking, Figure 11. A round bur was used under copious sterile irrigation to outline the cortical window within the cutting guide. With the guide removed, the margins of the planned osteotomy are deepened, and the outer 


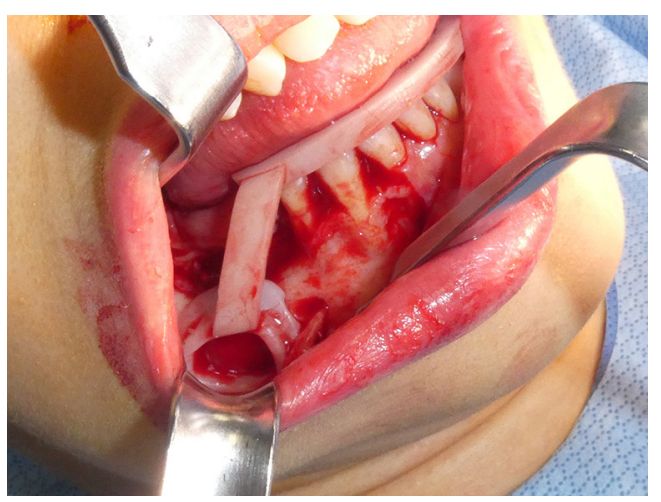

Figure 11 The fully seated guide intraoperatively with the exposed and retracted mental nerve.

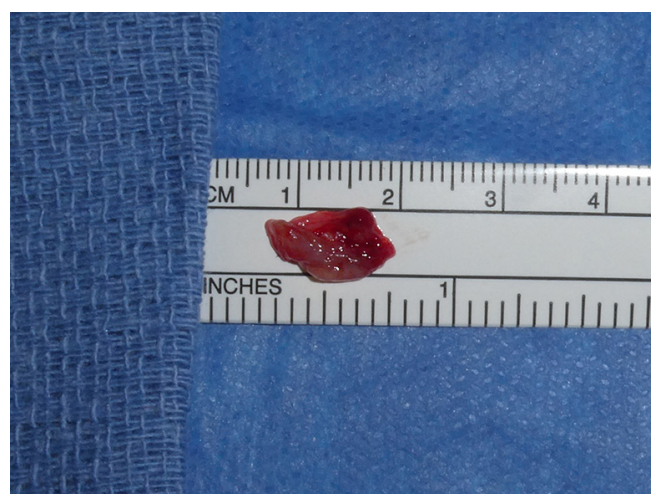

Figure 12 Recurrent ameloblastoma specimen removed in entirety.

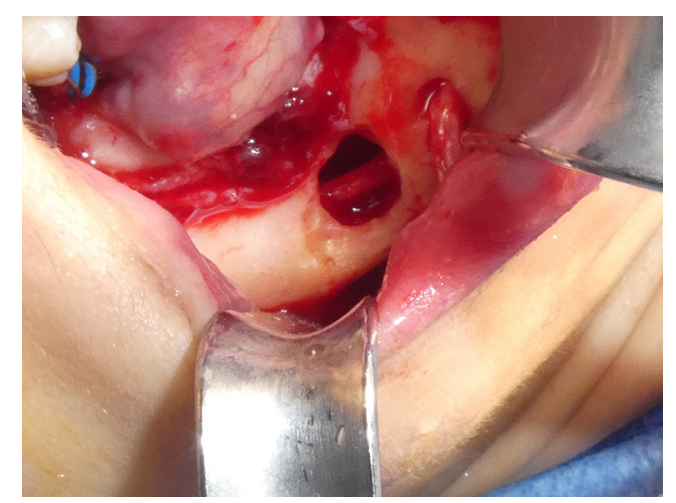

Figure 13 Intact inferior alveolar nerve is seen through the lateral window created with the aid of the surgical guide.

cortex was eventually removed to expose the margins of the underlying specimen. After identifying and then protecting the IAN within the cavity, the lesion was enucleated, Figure 12, followed by peripheral ostectomy and application

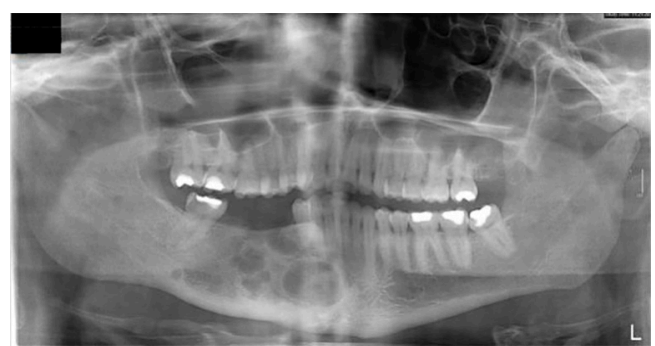

Figure 14 The panoramic image demonstrates multiple radiolucent lesions with radiopaque borders in the mandible.

of Carnoy's solution. Intact IAN can be seen in Figure 13 after excision of the recurrent lesion. On follow up, the patient showed no signs of V3 paresthesia and had an uneventful post-operative course.

\section{Case presentation 3}

A 34-year-old female presented to the hospital clinic with recurrent lesions of her right mandible. The patient presented with history of unknown surgical treatment and diagnosis in her home country. She had no other relevant medical history, and her review of systems was unremarkable. Clinically, she had an expansile lesion of her right mandible with pain and some degree of V3 paresthesia. On panoramic image, Figure 14, there are multiple radiolucent lesions with radiopaque borders within the mandibular body and extending toward the symphysis. An incisional biopsy was completed under intravenous sedation and local anesthesia, which resulted in diagnosis as ameloblastoma. The biopsy results and surgical options were again discussed with the patient. Especially in light of the significant size of her recurrence, it was recommended that she undergo definitive treatment of resection, however, the patient would not accept this modality. She opted for a conservative approach with close post-operative follow up for recurrences, as it had been performed previously.

CT maxillofacial exam, Figures 15,16, revealed multiple radiolucent lesions within the mandibular body, one of which was expansile in nature and exceeding the buccal and lingual boundaries of the anatomic mandible. The DICOM data from the patient's preoperative CT scan was utilized in a VSP session to design two cutting custom guides to access the multiple intramandibular lesions necessitating treatment (Figures 17,18). Of note, an intraoral scan was not required in the design of this occlusion and bone borne surgical guide due to availability. Similar surgical procedure 


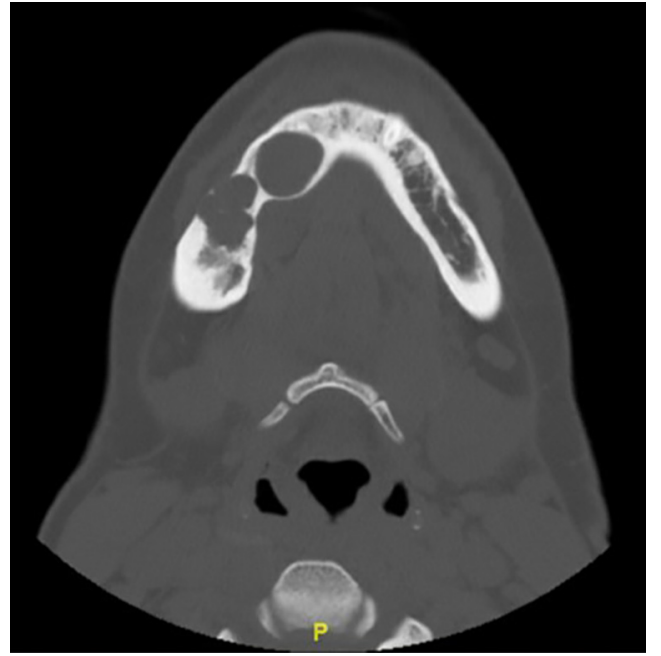

Figure 15 The axial view of the preoperative CT Maxillofacial exam allows for appreciation of the expansile nature of the patient's multiple lesions.

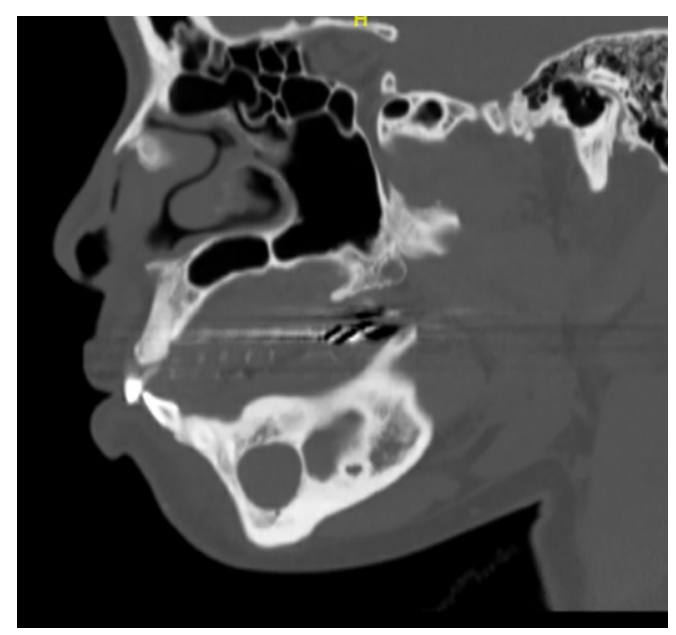

Figure 16 Multiple radiolucent lesions are appreciated again on the sagittal view of the patient's CT.

as described in the previous case presentations was followed, which is appreciated in Figures 19,20. No difficulty or discrepancy in the fit and stability of the guide was noted intraoperatively. The procedure was again completed with the patient's informed consent and without complications. The patient's post-operative course has been uneventful and no recurrences have bene appreciated on postoperative panoramic images thus far.

All procedures performed in this study were in accordance with the ethical standards of the institutional

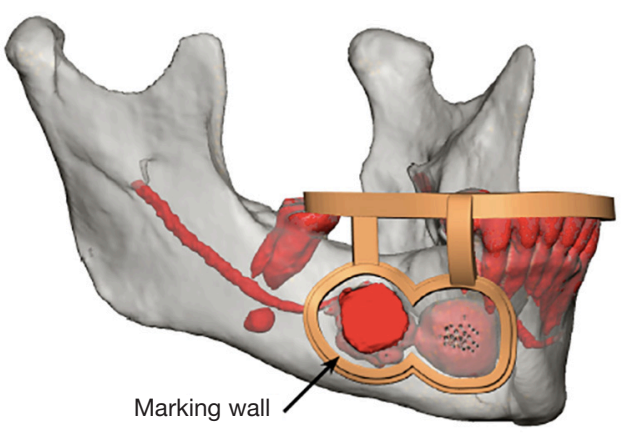

Figure 17 Occlusal and bone borne surgical guide design demonstrating the access to the anterior recurrent mandibular lesions.

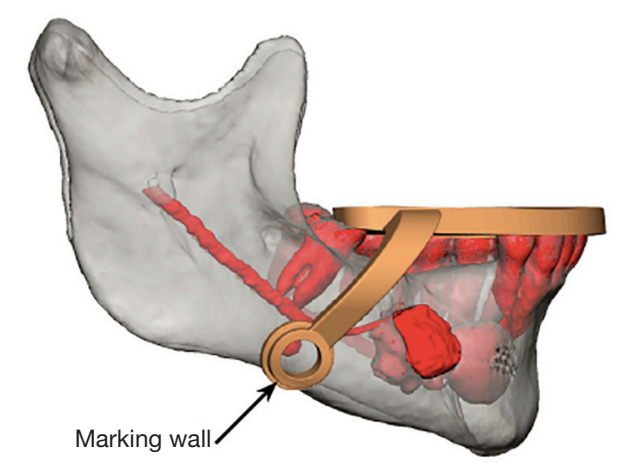

Figure 18 A second surgical guide was designed to access a smaller site of recurrence in the posterior mandible.

and/or national research committee(s) and with the Helsinki Declaration (as revised in 2013). Written informed consent was obtained from the patients for publication of this case report and accompanying images. A copy of the written consent is available for review by the editorial office of this journal.

\section{Discussion}

Accessing lesions without a surgical guide can lead to unnecessarily large osteotomies and incision sizes as the surgeon is responsible for translating the findings from the CT scan to the surgical field without any guidance. This inherently possesses an increased risk for iatrogenic injury and surgical trauma, potentially resulting in more post-operative edema, inflammation and pain (2). Though a CT scan is beneficial in understanding the location and 


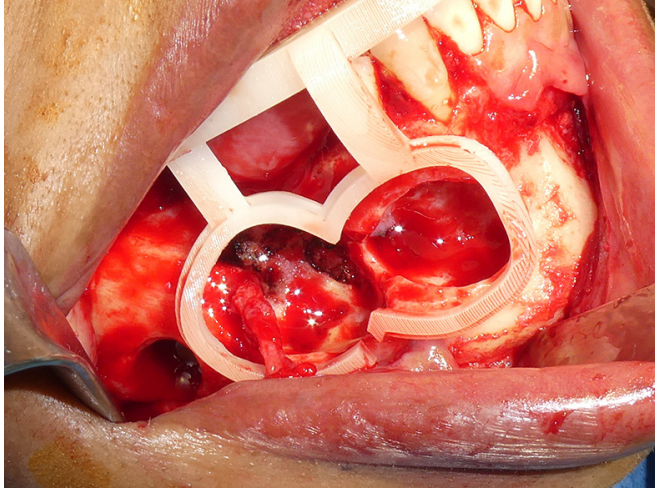

Figure 19 The osteotomy has been created with removal of lateral window from the anterior sites allowing for exposure of the intramandibular lesions.

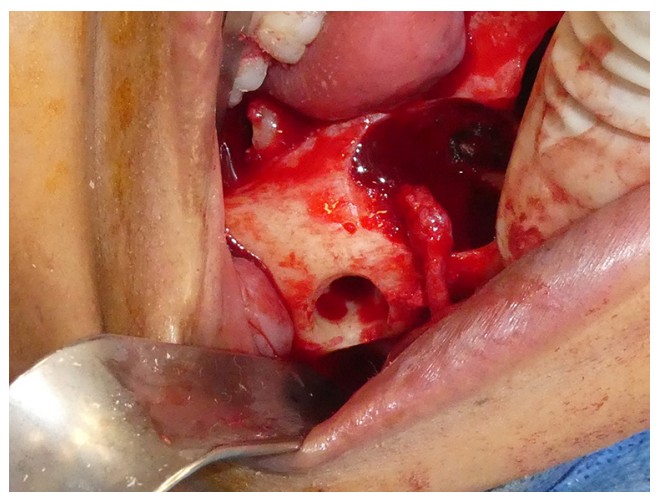

Figure 20 The surgical guides have been removed and the posterior osteotomy can be appreciated.

anatomical boundary and relationship of the lesion with nearby structures, such as the IAN, utilizing a surgical guide can potentially greatly reduce surgical morbidity.

Additionally, when a patient requires exploratory microsurgery during which the IAN must be accessed, a surgical guide has significant benefits when creating the osteotomy. Another method to access the IAN for exploratory microsurgery and other procedures, such as accessing cystic lesions and impacted teeth, other than a lateral window approach is a sagittal split osteotomy. This technique includes its own set of risks in both the nerve being accessing and post operative complications, such as hardware failure, malunion, or malocclusion $(8,9)$.

When a procedure is anatomically involved with nearby vital structures, preservation of these structures may limit surgical success. Performing surgical procedures as minimally invasive as possible may decrease surgical time and length of general anesthesia, decrease healthcare costs, and decrease surgical trauma and potential morbidity $(1,3-6)$. The use of surgical guides is highly variable and adaptable to surgical needs. In this series, the guides were useful in honing surgical accuracy to perfect the location of the created osteotomy. It may be beneficial in some circumstances to incorporate a depth gauge or drill stop to the VSP guide in order to control the depth at which the bur can penetrate. Custom guide fabrication utilizing data from CT imaging boasts a wide array of applications and are now considered a mainstay in craniomaxillofacial surgeries, from peripheral ostectomy and resection of benign and malignant pathology of the mandible respectively, reconstruction with free vascular flaps, facial trauma reconstruction, orthognathic surgery and implant dentistry $(1,7,10)$.

\section{Conclusions}

Utilizing VSP technology to design and fabricate custom surgical guides with patient's pre-operative imaging data can be beneficial in a multitude of ways. Aiding in the operation of a more precise and efficient surgery, surgical guides may decrease morbidities and may also potentially bolster patient outcomes by minimizing complications. Surgical guides can be implemented in an array of surgeries, from trauma, pathology, microsurgery, implant surgery, and correction of dentofacial and skeletal deformities.

\section{Acknowledgments}

Funding: None.

\section{Footnote}

Provenance and Peer Review: This article was commissioned by the Guest Editors (Shahid Aziz and Pooja Gangwani) for the series "Virtual Surgical Planning in Maxillofacial Surgery" published in Frontiers of Oral and Maxillofacial Medicine. The article has undergone external peer review.

Peer Review File: Available at https://fomm.amegroups.com/ article/view/10.21037/fomm-21-84/prf 
Reporting Checklist: The authors have completed the CARE reporting checklist. Available at https://fomm.amegroups. com/article/view/10.21037/fomm-21-84/rc

Conflicts of Interest: Both authors have completed the ICMJE uniform disclosure form (available at https://fomm. amegroups.com/article/view/10.21037/fomm-21-84/coif). The series "Virtual Surgical Planning in Maxillofacial Surgery" was commissioned by the editorial office without any funding or sponsorship. VZ serves as a consultant for Axogen, Inc (Alachua, FL); and serves as an unpaid editorial board member of Frontiers of Oral and Maxillofacial Medicine from November 2019 to October 2021. The authors have no other conflicts of interest to declare.

Ethical Statement: The authors are accountable for all aspects of the work in ensuring that questions related to the accuracy or integrity of any part of the work are appropriately investigated and resolved. All procedures performed in this study were in accordance with the ethical standards of the institutional and/or national research committee(s) and with the Helsinki Declaration (as revised in 2013). Written informed consent was obtained from the patients for publication of this case report and accompanying images. A copy of the written consent is available for review by the editorial office of this journal.

Open Access Statement: This is an Open Access article distributed in accordance with the Creative Commons Attribution-NonCommercial-NoDerivs 4.0 International License (CC BY-NC-ND 4.0), which permits the noncommercial replication and distribution of the article with the strict proviso that no changes or edits are made and the original work is properly cited (including links to both the formal publication through the relevant DOI and the license). See: https://creativecommons.org/licenses/by-nc-nd/4.0/.

doi: 10.21037/fomm-21-84

Cite this article as: Gory C, Ziccardi V. Virtual surgical planning of inferior alveolar nerve microsurgery and benign pathology of the mandible: case reports. Front Oral Maxillofac Med 2022;4:30.

\section{References}

1. Mehra P, Miner J, D'Innocenzo R, et al. Use of 3-d stereolithographic models in oral and maxillofacial surgery. J Maxillofac Oral Surg 2011;10:6-13.

2. Cohen JM, Ziccardi VB. Use of Virtual Surgical Planning as an Adjunct for Enucleation of Multiple Recurrent Odontogenic Keratocysts: Case Report. J Oral Maxillofac Surg 2018;76:2137.e1-6.

3. Diment LE, Thompson MS, Bergmann JHM. Clinical efficacy and effectiveness of 3D printing: a systematic review. BMJ Open 2017;7:e016891.

4. Serrano C, Fontenay S, van den Brink H, et al. Evaluation of $3 \mathrm{D}$ printing costs in surgery: a systematic review. Int J Technol Assess Health Care 2020. [Epub ahead of print].

5. Resnick CM, Inverso G, Wrzosek M, et al. Is There a Difference in Cost Between Standard and Virtual Surgical Planning for Orthognathic Surgery? J Oral Maxillofac Surg 2016;74:1827-33.

6. Ballard DH, Mills P, Duszak R Jr, et al. Medical 3D Printing Cost-Savings in Orthopedic and Maxillofacial Surgery: Cost Analysis of Operating Room Time Saved with 3D Printed Anatomic Models and Surgical Guides. Acad Radiol 2020;27:1103-13.

7. Ganz SD. Techniques for the use of CT imaging for the fabrication of surgical guides. Atlas Oral Maxillofac Surg Clin North Am 2006;14:75-97.

8. de Oliveira JC, Garcia IR Jr, de Melo WM, et al. Sagittal mandibular osteotomy for removal of intraosseous lesion. J Craniofac Surg 2014;25:e237-9.

9. Rittersma J, van Gool AV. Surgical access to multicystic lesions, by sagittal splitting of the lower jaw. J Maxillofac Surg 1979;7:246-50.

10. Tack P, Victor J, Gemmel P, et al. 3D-printing techniques in a medical setting: a systematic literature review. Biomed Eng Online 2016;15:115. 